\title{
Enhancing Fault Isolation for Health Monitoring of Electric Aircraft Propulsion by Embedding Failure Mode and Effect Analysis into Bayesian Networks
}

\author{
Chetan S. Kulkarni ${ }^{1}$, Matteo Corbetta ${ }^{2}$, and Elinirina I. Robinson ${ }^{3}$ \\ 1, 2,3 KBR Inc., NASA Ames Research Center, Moffett Field, CA, 94035, USA \\ chetan.s.kulkarni@nasa.gov \\ matteo.corbetta@nasa.gov \\ elinirina.robinson@nasa.gov
}

\begin{abstract}
This paper describes a fault isolation approach for electric powertrains of unmanned aerial vehicles. The approach leverages the combination of failure mode and effect analysis (FMEA) and Bayesian networks, thus introducing dependability structures into a diagnostic framework. Faults and failure events from the FMEA are mapped within a Bayesian network, where network edges replicate the links embedded within FMEAs. This framework helps the fault isolation process by identifying the probability of occurrence of specific faults or root causes given evidence observed through sensor signals. The framework is applied to an electric powertrain system of a small, rotary-wing unmanned aerial vehicle, demonstrating how a Bayesian network enhanced by FMEA helps disambiguate between root causes of incipient failures, which would otherwise be considered as equally probable.
\end{abstract}

\section{INTRODUCTION}

Electric powertrains are at the forefront of the aeronautics transformation (Raymer, 2018), (NASA, 2015), (Larson, 2015), (Rosero, Ortega, Aldabas, \& Romeral, 2007). They are expected to propel a variety of unmanned aerial vehicles in the low-altitude airspace, from small drones for package delivery to larger, on-demand, urban air mobility-type of vehicles. Autonomous, electrically-powered VTOL (vertical take off and landing) vehicles are among the best candidates to fulfill the new set of low-altitude airspace requirements based on space constraints as well as operational benefits.

The foreseeable high traffic density suggests that a large number of these electric propulsion systems will enter the airspace, and that they will also operate at high frequency, e.g., large number of take offs and landings per unit time.

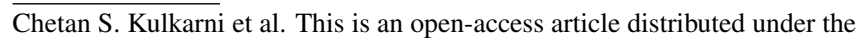
terms of the Creative Commons Attribution 3.0 United States License, which permits unrestricted use, distribution, and reproduction in any medium, provided the original author and source are credited.
}

The reliability of such critical systems is therefore key to ensure high safety standards in the low-altitude airspace. Diagnostic systems, which aim at identifying incipient faults, can mitigate unexpected failures or lower-than-expected reliability by performing early fault detection using sensor data. A key element of fault diagnosis is fault detection and isolation (FDI), which complexity increases with the complexity of the system itself, namely the number of subsystems and components, interactions among sub-systems, and the number of sensors available. System diagnosis is a fundamental step to further prognosticate the remaining useful life of a subsystem, trigger mitigating actions during flight or request maintenance actions in-between flights.

This work merges information from the electric propulsion system design phase with diagnostic tools, which are often developed later in the product lifecycle, or retrofitted to existing specimens. Information from the failure mode and effect analysis (FMEA) from the system design phase is embedded within a Bayesian network (BN) (Pearl, 1985). Each node in the network can represent either a fault, failure mode, root cause or effect, and the causal relationships between different elements are described through the connecting edges. The approach demonstrates that FMEAs can aid diagnostics, prognostics and health management by feeding causal relationships identified during the design phase into a diagnostic framework. Earlier works in this area can be found in (Lee, 2001), where the idea of mapping FMEA into BNs was developed, and (Bobbio, Portinale, Minichino, \& Ciancamerla, 2001), where a failure tree was embedded in $\mathrm{BN}$.

Earlier research work in the area of autonomous system diagnosis was presented in (Kulkarni, Celaya, Biswas, \& Goebel, 2012; Daigle, Sankararaman, \& Kulkarni, 2015), which focused on individual systems and components to implement prognostics methodologies. In the later approaches, effects of component-level degradation on the system as a whole were studied to implement the prognostics framework 


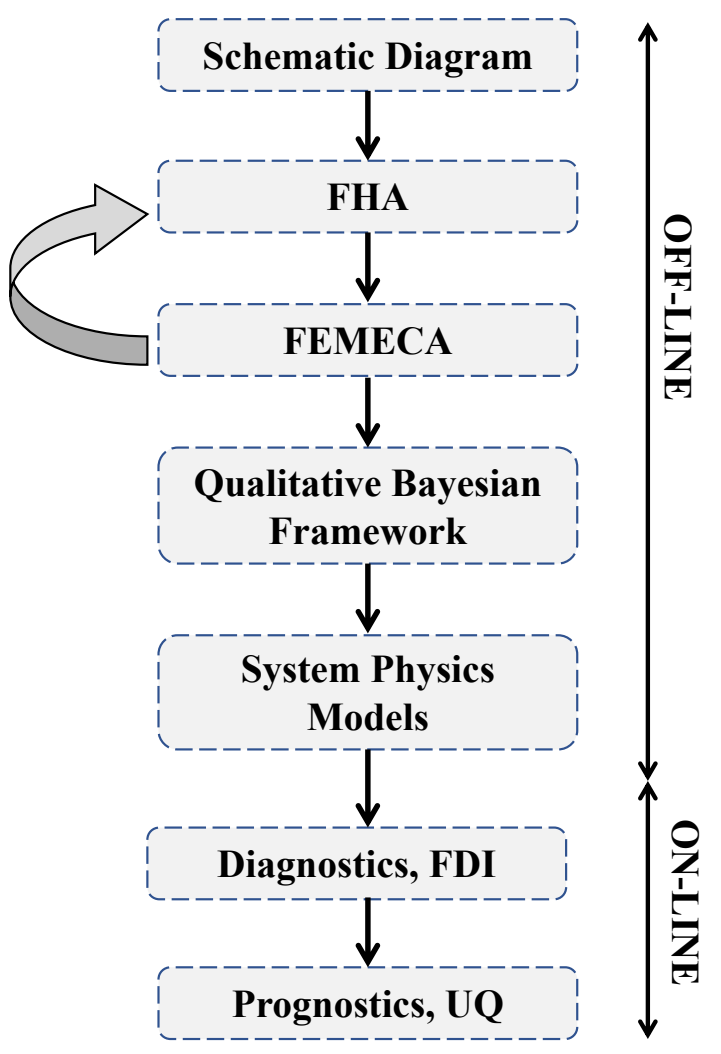

Figure 1. Process Flow Chart of the implemented approach to UAV Health Management

(G. Gorospe, Kulkarni, \& Hogge, 2017; G. E. Gorospe \& Kulkarni, 2017). The development of new models and integration with previous models enables us to study and identify cascaded effects of degradation on connected power-train systems during operation. In (Hogge et al., 2018), the implementation of a prognostic framework to batteries in fixedwing e-UAS was studied. The process flow in Figure 1 shows the implementation of the integrated FMEA and Qualitative Bayesian approach, and where the FDI methodology discussed in this work stands in the health monitoring framework proposed in those previous works ${ }^{1}$.

This work differs from earlier attempts where BNs aided the design of the FMEA structure (Lee, 2001), and from (Bobbio et al., 2001), where dependability between system components were defined by failure trees. Here, the BN supports FDI, possibly in real time as new sensor data are collected during or post-flight capturing system aging and degradation to update failure probabilities. The specificity of the electric powertrain health monitoring system also require further adaptation of previous works. The paper discusses issues related to the embedding of probability of fault detection, false alarms, and other indicators of system diagnosis performance into the framework.

\footnotetext{
${ }^{1}$ The acronyms in Figure 1 not yet defined will be defined later in the section.
}

The paper's goal are two-fold. First, present how the developed approach can help FDI, discussing issues like probability of mis-detection, false alarms, and sensor sensitivity in compiling the conditional probability tables. Second, embedding of FMEA information into a BN for a specific case study, namely the powertrain system of a small e-UAV. The result is a framework capable of isolating the cause of sub-system level fault and degradation.

Our broader research effort is to identify failure modes, develop a failure modes and effects criticality analyses (FMECA) for the system and combine it within a fault detection and prognostics framework. This research is aimed towards accomplishing the following objectives:

- Identify and quantify the effects of the identified hazards, the severity and probability of their effects, their root cause and the likelihood of each cause.

- Develop a Bayesian framework for fault detection and isolation

- Based on the FDI output estimate health of the faulty component and predict the remaining useful life (RUL), by also performing uncertainty quantification (UQ) for all the steps of the process.

- To identify potential electric powertrain hazards and perform a functional hazard analysis (FHA) for unmanned aerial vehicles (UAVs)/ Urban Air Mobility (UAM) vehicles.

\section{BACKGROUND}

The need for safety assurance and health management capabilities is particularly relevant for aircraft electric propulsion systems, which are relatively new and with limited (or none) historical data to learn from. They are critical systems requiring high power density along with reliability, resilience, efficient management of weight, and operational costs. It is clear how a fault diagnosis and prognosis tool can aid such safety and reliability requirements, as well as system stateawareness, thus enabling autonomous decision making capabilities for safe and efficient operation. The approach proposed in this paper allows to compute in quantitative ways the failure probabilities of the components (or sub-systems) of such complex systems, while integrating modeling and measurement uncertainty.

\subsection{Failure Mode and Effect Analysis}

Failure Mode and Effect Analysis (FMEA) methodology has evolved over the years, introducing variations like process FMEA, design FMEA, FMEA embedding criticality analysis (failure modes, effects, and criticality analysis, FMECA), etc. In this work a general FMEA methodology is being implemented. In reference to the general definition SAE J1739 (SAE, 2015), the scope is narrowed here, with the key ob- 
jective of FMEAs to recognize and evaluate potential failures and their effects, identify the weak links within the system (i.e., powertrain) that leads to failures, and develop resources to reduce the chance of potential failure occurring at those links. The FMEA will aid the diagnostic process by providing the causal links among failure events potentially occurring within the system.

The list below shows an FMEA header example. Such a list is not always exhaustive, and subject to changes based on particular component, system, application etc.

- Failure / degradation mode

- Failure effect

- Severity

- Cause

- Frequency

- Detectability

- Risk Priority Number

The FMEA table developed is based on this header example but curated for a powertrain of a quadrotor electric vehicle discussed later in the Section 3. Functions were defined at the equipment level to facilitate the development of meaningful functional failures. Each function was reviewed and potential failure modes were postulated for each function and assigned failure mode.

Failure rates depend and vary based on design, application, and operation of the system. Reliability is designed into equipment based on operational requirements, which is developed by the manufacturer based on resulting consequences observed in terms of severity or criticality due to failure. In this study, some of the failure rates were obtained through the manufacturer of the subsystems, while some others were defined based on similarities to other systems. In the future, once the actual failure rates are obtained, the tool will be able to generate an updated output.

Currently, state-of-the-art components designed for specific distributed electric/hybrid-electric propulsion vehicles may not have accurate reliability data based on operations (Darmstadt \& et al, 2019). This gap is currently being filled thanks to some parallel work (Kulkarni et al., 2017), where a hardware-in-loop testbed in being developed to test and age the components. Some of our earlier work included Virtual lab (Kulkarni et al., 2017) where powertrain components were loaded using realtime data from the UAV vehicle to age the subsystems. Once the system is fielded in the testbed, aging tests are performed using accelerated aging methods, and the system is tracked to keep records of any degradation due to operational loads. The data from which is then used to update the degradation/failure rates of respective sub-system.
FMEAs may not cover all possible fault modes when systems become very complex. Hence, for such systems, approaches to combine FMEA and FTA are being implemented (Cristea \& Constantinescu, 2017). FTA is a top-down analysis approach which captures interrelationships between respective subsystems of the powertrain. The FTA documents any catastrophic or severe outcome at the top level, while the less severity hazards become evident based on the FTA structure that describes the subsystems interrelationship with each other. The UAV propulsion system consists in the electronics that control the motors, the motors, and batteries, all captured in the FTA. The top level hazards defined from the propulsion system FHA were used to inform the top level of the fault tree. The FMEA combined with details from FTA is evolved further using an Bayesian framework which will be discussed in the later section.

\subsection{Bayesian Networks}

Bayesian Networks (BNs) are directed acyclic graphs where nodes represent propositions or variables, the arcs represent the existence of direct causal influences between the linked propositions, and the strength of the causal relationships is represented through conditional probabilities (Pearl, 1985). The example below, reproduced from (Pearl, 1985), is used as illustrative explanation of how $\mathrm{BN}$ works.

Figure 2 shows a representative $\mathrm{BN}$, where the complete joint probability distribution $p\left(x_{1}, x_{2}, x_{3}, x_{4}, x_{5}, x_{6}\right)$ is the product of the conditional probabilities of each proposition given its ancestors, Eq. (1).

$$
\begin{aligned}
p\left(x_{1}, \ldots, x_{6}\right)= & p\left(x_{6} \mid x_{5}\right) p\left(x_{5} \mid x_{2}, x_{3}\right) p\left(x_{4} \mid x_{1}, x_{2}, x_{3}\right) \\
& p\left(x_{3} \mid x_{1}\right) p\left(x_{2} \mid x_{1}\right) p\left(x_{1}\right)
\end{aligned}
$$

The joint probability distribution could also be expressed

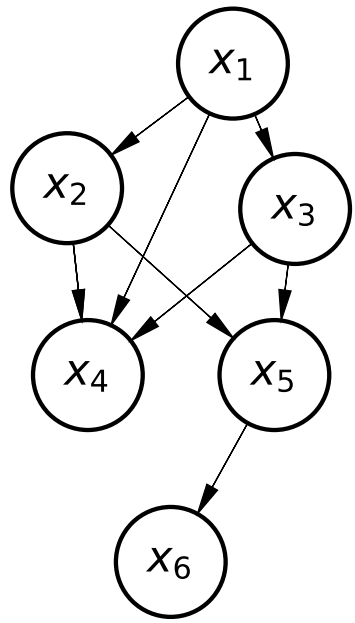

Figure 2. Example of directed acyclic graph used to create a Bayesian network. 
with the short notation:

$$
p(\boldsymbol{x})=\prod_{j=1}^{n} p\left(x_{j} \mid \boldsymbol{a}_{j}\right),
$$

where $\boldsymbol{a}_{j}$ represents the set of ancestors of variable $x_{j}$, and $\boldsymbol{x}$ is the random vector containing all variables $x_{1}, \ldots, x_{n}$ (Pearl, 2000; Bobbio et al., 2001). For example, the term $p\left(x_{4} \mid x_{1}, x_{2}, x_{3}\right)$ becomes $p\left(x_{4} \mid \boldsymbol{a}_{4}\right)$.

Dependencies among propositions are described through the definition of sets of ancestors (or parents) and descendants (or children). For example, the set $\left\{x_{1}, x_{2}, x_{3}\right\}$ contains the ancestors of $x_{4}$, while $\left\{x_{2}, x_{3}\right\}$ contains the children of $x_{1}$. This structural model allows analysis over interventions, i.e., enable the computation of the joint probability density function (pdf) conditioned on some specific assumptions over a specific variable in the network (Pearl, 2000). Starting from the example in Figure 2, it is possible to evaluate the joint pdf given, e.g., $x_{2}$ has been defined True:

$$
\begin{aligned}
p_{X_{2}=1}\left(x_{1}, x_{3}, \ldots, x_{6}\right)= & p\left(x_{6} \mid x_{5}\right) p\left(x_{5} \mid X_{2}=1, x_{3}\right) \\
& p\left(x_{4} \mid x_{1}, X_{2}=1, x_{3}\right) p\left(x_{3} \mid x_{1}\right) \\
& p\left(x_{1}\right) .
\end{aligned}
$$

The dependency of $x_{2}$ from $x_{1}$ has been removed in Eq. (3), since forcing $X_{2}=1$ does not depend on the value of $x_{1}$. Therefore, the edge connecting $x_{1}$ to $x_{2}$ should be removed to represent the proposed intervention. The challenge presented by $\mathrm{BN}$ is the assessment of all conditional probabilities of the system. Each node of the network requires a conditional probability table that defines the probability of the node being 1 (or 0 ) given all possible values of its ancestors. The dimension of the table increases in a combinatorial fashion with the number of ancestors. Some of these issues are addressed in Section 3 where a BN is applied to a electric propulsion system diagnosis.

\subsection{Moving from FMEA to $B N$}

In order to develop an efficient system level diagnosis procedure that takes uncertainty into account, both information from the FMEA and from the BN are combined. To this end, two main steps are involved: (i) build the $\mathrm{BN}$ structure and (ii) establish the conditional probability tables (CPT) of each node.

In order to construct the BN structure, the qualitative information about failure modes, causes and effects contained in the FMEA worksheet are transformed into nodes. As a starting point, observable nodes (simulating sensors), may represent simply events triggered by sensor signals, like, for example, a boolean variable for "temperature of the electronic speed controller too high". Such information can help disambiguate among potential causes of the observed event. Then, the relationships between each node is defined by pointing arcs from causes to failure modes, and from failure modes to effects, resulting in the construction of the $\mathrm{BN}$ structure. A simple example of a BN structure build from FMEA is shown in Fig.3 for illustration.

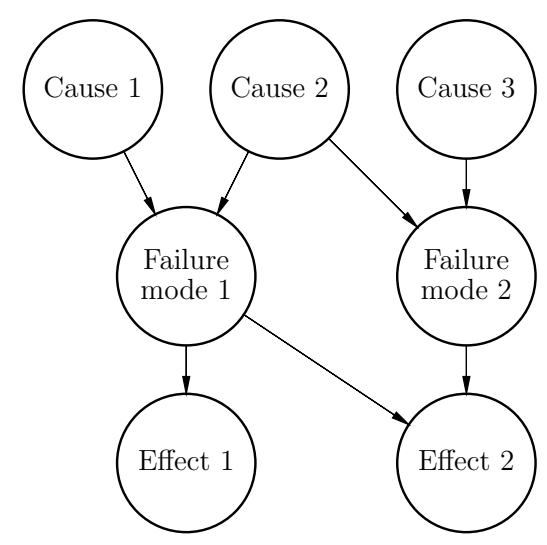

Figure 3. Example of a simple BN structure build from FMEA.

Once the BN structure is built, the next step consists in assigning prior marginal probabilities to the root nodes (with no ancestors) and conditional probabilities to each of the other nodes, based on the qualitative information provided by the FMEA.

Probability values can be defined for example from historical failure data, expert knowledge about the probability of failure of each component, or by using maximum entropy theory as in (Gilabert, 2011).

Once the BN is completely defined, it can be used to detect and localize a fault within a complex system by turning observable nodes to True or False. The diagnosis procedure updates the probabilities using Bayesian inference in order to determine the root cause with the highest failure probability when an evidence (observable) node is triggered. The evidence nodes, also known as fault symptoms, are associated to observed variables such as sensor measurements, and can be for example triggered when the observed value exceeds a certain threshold.

Possible external sources that can affect the diagnosis procedure, such as environmental conditions or false alarm, are discussed in the next section.

\subsection{Modeling approach and issues}

The dependency among elements in FMEAs do not have to be restricted to deterministic relationships in BNs (Bobbio et al., 2001), and this property intrinsically enhances the modeling of the diagnostic system. Let us consider, for simplic- 
Table 1. Example of conditional probability table for a fault event with two known root causes.

\begin{tabular}{cccc}
\hline$x_{1}$ & $x_{2}$ & \multicolumn{2}{c}{$f$} \\
& & 0 & 1 \\
\hline 0 & 0 & $p_{0 \mid 00}$ & $p_{1 \mid 00}$ \\
0 & 1 & $p_{0 \mid 01}$ & $p_{1 \mid 01}$ \\
1 & 0 & $p_{0 \mid 10}$ & $p_{1 \mid 10}$ \\
1 & 1 & $p_{0 \mid 11}$ & $p_{1 \mid 11}$ \\
\hline
\end{tabular}

ity, a fault event $f$ with two root causes, its ancestors, $x_{1}$ and $x_{2}$. Table 1 is the conditional probability table of the model, where probabilities are defined through three binary subscripts $i, j, k \in\{0,1\}$. The term $p_{k \mid i j}$ defines the probability of the outcome $k$ given values $i, j$, with $k$ referring to the fault event $f$ and $i, j$ referring to its ancestors $x_{1}$ and $x_{2}$. For example, $p_{1 \mid 00}$ is the probability that $f=1$ given both ancestors $x_{1}, x_{2}$ are 0 (or False).

The fault event may happen, with low probability, because of external causes or unknown events not described by its ancestors. Such external forcing was called Common Cause Failures in (Bobbio et al., 2001), and following that idea, $p_{1 \mid 00} \geq 0$, and so $p_{0 \mid 00}=1-p_{1 \mid 00}$. On the opposite side of the spectrum, the fault event may not happen even if both ancestors are activated (true). This option describes the ability of a system to work partially or reconfigure, (Bobbio et al., 2001), or describes a statistical relationship between the three elements, suggesting that root causes do not deterministically trigger the failure, so $p_{1 \mid 11}<1$. As a result, the two ancestors may occur without triggering the fault event, so $p_{0 \mid 11} \geq 0$ and $p_{1 \mid 11}=1-p_{0 \mid 11}$. Different ancestors may influence the fault event in different ways, e.g. according to the severity of the root cause. This properties can be easily embedded in the network by assigning different values to the probabilities conditioned over $\left\{X_{1}=1, X_{2}=0\right\}$ and $\left\{X_{1}=0, X_{2}=1\right\}$.

In addition to the cases of failures induced by external variables or prevented system reconfiguration, the BN should also account for the performance of the measuring and/or detection system. In the proposed architecture, the evidence used to perform inference over the network is collected through sensors that measure variables connected (directly or indirectly) to the fault event we aim to detect. The sensor performance or, similarly, the ability of the detection system to identify anomalous sensor data, should be embeeded in the estimation of the CPT values. Reconnecting to the previous example, therefore, the element $p_{0 \mid 00}$ in Table 1 should account for false alarm rates, and $p_{1 \mid 11}$ should include, on top of any statistical relationship between the elements, the probability of misdetection.

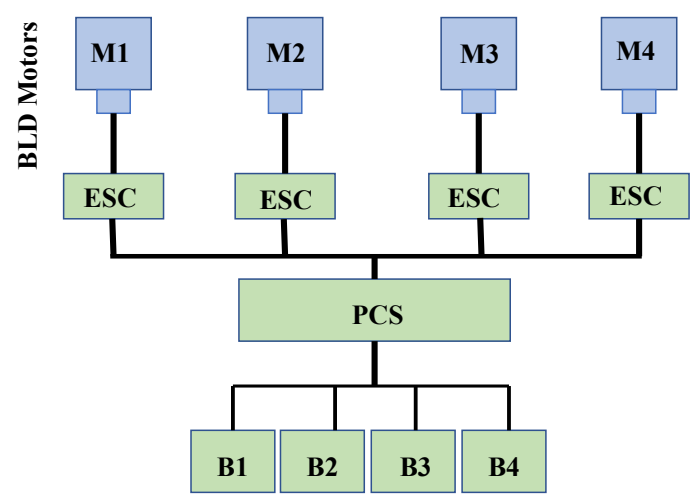

Figure 4. Schematic of basic quadcopter powertrain elements.

\section{ELECTRIC PROPULSION CASE STUDY}

This section shows how the BN-FMEA can aid the fault isolation process for a sub-system of the powertrain of a small autonomous rotorcraft by showing a simple, yet representative, working example. The powertrain design consists of a Li-ion battery composed of Li battery packs, power condition unit, electronic speed controller (ESC) with pulse-width modulation (PWM), and brushless DC motor. A scheme of the powertrain is shown in Figure 4.

From a practical standpoint, the first step is to build a FHA to assess any associated risk of failure in the system. The output from the hazard analysis is an assessment of the different type of hazards along with their probability of failures. Then, the analysis can move to FMEA/FMECA to include reliability and performance information, as well as the effect of faults and non-catastrophic failures.

The knowledge of prior probabilities typically comes from operational data, OEM data-sheets, and SME's to develop the framework. However, the probability of occurrence of failures from the FHA and FMEA can be qualitative, if historical data are not available, and that is the approach utilized in this work, to exemplify the methodology.

Bayesian theorem, at the foundation of BNs, can be implemented using quantitative as well as qualitative methods (Humphreys \& Jacobs, 2015; Medow \& Lucey, 2011), and so it is particularly suitable for the approach utilized here.

A simplified FMEA for a UAV vehicle currently used for our experiments is shown in Table. 2. This is a dynamic working table and is being updated as additional information becomes available from either the operation tests or SME's. The examples of BN application shown in the later subsections are based on the information from such table. The table is used to compute the qualitative conditional probability of occurrence of each element of the powertrain in the case sutdy. 
Table 2. FMEA for quadrotor e-UAS power-train system

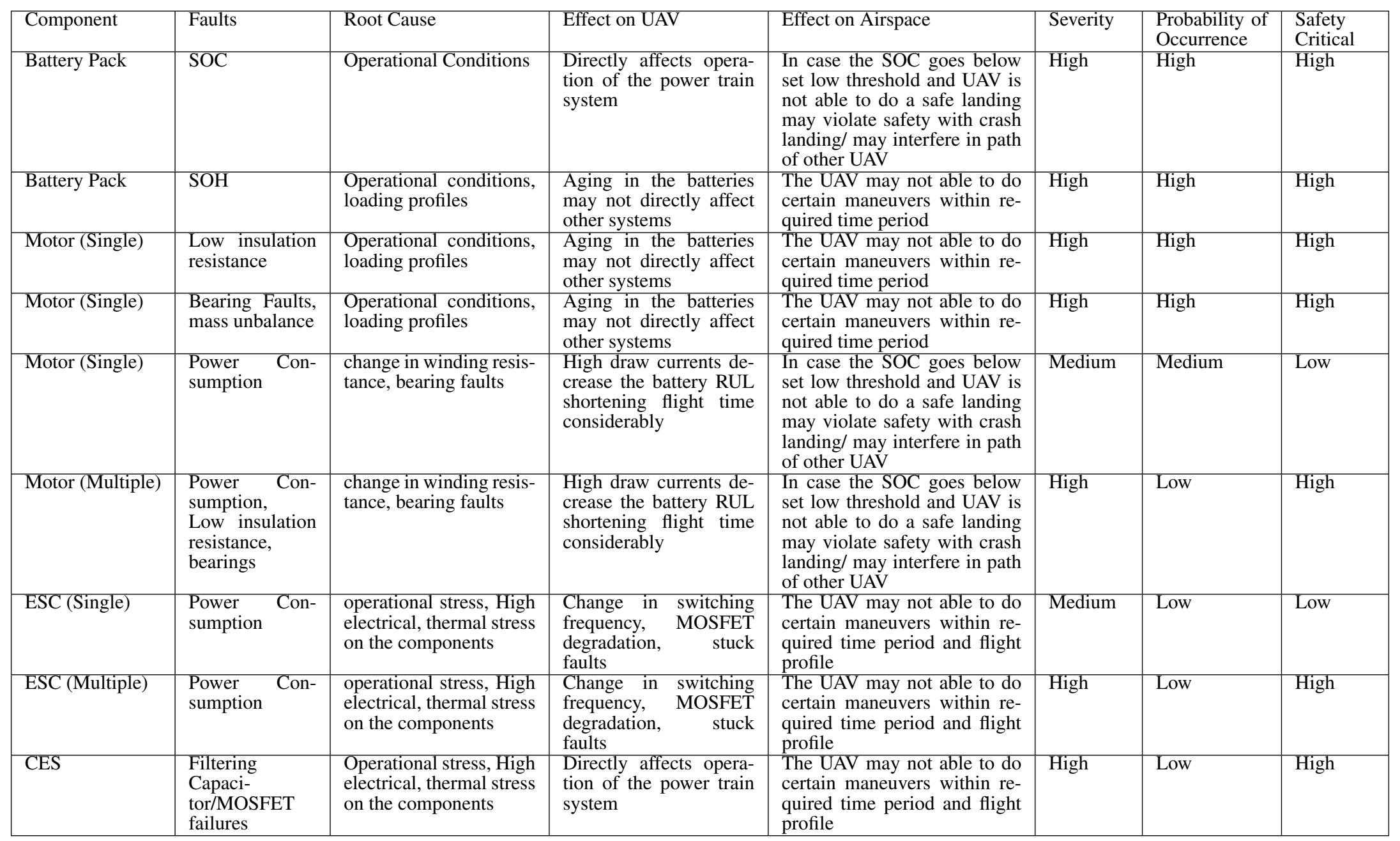


If the qualitative probability is in one of the intermediate categories, then further analysis is required and identified by the fault diagnosis framework. Further, once the fault is detected and isolated using the BN-based tool, the prognostics tool can be triggered to estimate and predict the RUL for the faulty sub-system. In this case the remaining useful time is defined as the time till the state of charge (SOC) of a battery pack reaches a lower bound threshold. In the next section an example is discussed which includes a few components from Table 2 for demonstration of the framework.

\subsection{Case Study Scenarios}

In this Subsection, different scenarios of faults observed in each of the sub-systems is discussed. These are based on the operational load conditions for the vehicle and may occur in flight. In the later section each fault case considered is then further used in the Bayesian formulation.

\subsubsection{Batteries}

Under nominal conditions the battery voltage is above a certain threshold during which time the vehicle is able to perform all required objectives which are updated prior as part of the flight plan.

Lithium corrosion, plating, electrolyte layer formation, and contact losses are examples of faults that batteries are susceptible to (Daigle \& Kulkarni, 2013). These faults lead to an increase in internal resistance and impedance, as well as a decrease in charge capacity. These are related to the stateof-health (SOH) of the battery. Based on vehicle and safety requirements, the SOC threshold is set such that the vehicle must be capable to make a safe landing. Nonetheless, certain faults may lead to the battery discharging at a faster rate. This could be due to some internal battery pack fault or due to other connected subsystems down the stream. One of the scenarios considered is a large resistive fault in the wiring which may discharge one of the battery packs faster and loose SOC considerably. The faults described here will be at the foundation of the BN utilized to perform FDI.

\subsubsection{Electronics Speed Controller}

Electronics speed controllers (ESCs) are half bridge rectifiers and most of the faults observed in them are due to switching circuits. Faults in the conditioning circuit are not considered here. Such switching-circuit faults are observed when the MOSFETs are not synchronized while operating, or when the switching circuit is malfunctioning (G. Gorospe et al., 2017). The first one results in a variable PWM control waveform, and the second one results in a non-operational voltage phase switching pair. Generally, a degraded ESC or an ESC operating under a faulty condition will draw more/less current than a healthy ESC when operating under similar environmental conditions and load.

\subsubsection{BLDC Motor}

DC motors are susceptible to mechanical faults in the form of general motor or bearing wear, and electrical faults in the form of poor contacts and insulation deterioration (Abramov, Nikitin, Abramov, Sosnovichellasosnovich, \& Bozek, 2014; Awadallah \& Morcos, 2002). Typically, changes in the vibration characteristics are caused by mechanical faults, and changes in the current draw characteristics are caused by electrical faults, but can also be caused by mechanical degradation. For example, bearing wear can result in increased friction, which would result in higher current draw to maintain the same output due to the increase in mechanical resistance.

In addition, winding degradation over time, due to high load usage, cause changes in insulation resistance. Winding faults can lead a short or open circuit condition.

\subsection{Computing conditional probabilities given sensor- based fault detection}

In this section, the fault diagnosis procedure presented earlier in Section 2.3 is applied to the fault scenarios described in Section 3.1.

First, based on the FMEA in Table 2, the BN architecture of the case study is developed. As can be seen in Figure 5, different colors distinguish the main components; battery nodes are in blue, motor nodes in red and ESC nodes in pear green. The root cause nodes in black represent chemical reaction in the battery (chem), flight profile, operational conditions (op. cond), and electrical/thermal stresses (el. therm stress). As described in the previous subsection, the failure mode nodes are composed of: (i) three battery faults, SOC, SOH and thermal runaway (therm. runaway), (ii) two ESC faults, MOSFET and PWM, and (iii) two motor faults, bearing and winding resistance (winding res.). Finally, the observation nodes for each of the three components are related to their voltage, current and temperature measurement sensors; battery voltage $V_{b}$, current $I_{b}$, and temperature $T_{b}$, ESC voltage $V_{e}$, current $I_{e}$, and temperature $T_{e}$, and motor voltage $V_{m}$, current $I_{m}$, and temperature, $I_{m}$.

The architecture shows the causal relationships between the nodes, but it is necessary to quantify such relationships by assigning all node prior probabilities conditioned on the state of their ancestors. Despite the limited size of the problem considered, the solution requires the definition of several CPTs. The prior conditional probabilities utilized to produce the results are reported in Appendix 5. They are based on qualitative assumptions by subject matter experts, are for illustration only and should not be considered accurate. Indeed, as more information about the system failure probabilities are available over operational usage, these values can be updated for more accuracy. The prior probability of root causes like operational conditions or flight profile (black nodes) are all set to 


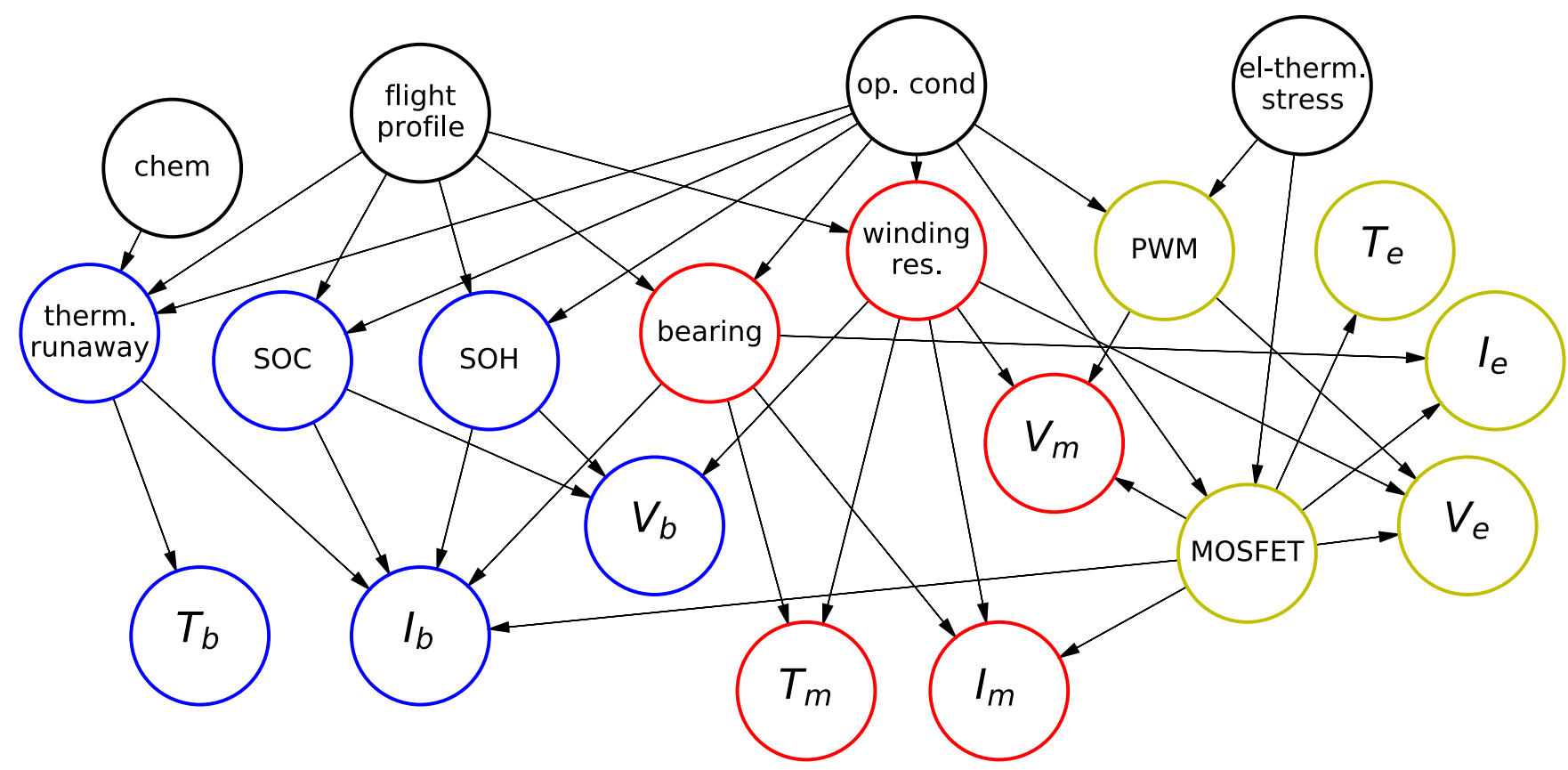

Figure 5. Bayesian network of the powertrain case study

default values of $1 e-6$, since those are dependent on the true operations of the vehicle. Also, assessing such causes, for example whether the ambient temperature is high with respect to the operational range of the battery, is straightforward, and the corresponding nodes could be set to True to assess how much such external factors influence the isolation process.

Once the prior probability values are assigned to each of the nodes, the constructed $\mathrm{BN}$ can be used for fault isolation. Indeed, when abnormal sensor measurements are detected, the BN will update the failure probability values of the fault nodes. This will allow the isolation of the most probable faulty component, which is the one with the highest failure probability given the abnormal sensor measurements. The proposed diagnosis methodology in this paper is based only on the multiple sensor measurements, and enables the disambiguation of the causes. For simplicity, performance of sensors or anomaly detection systems is not discussed, and implicitly embedded in the conditional probability tables in Appendix 5. However, the reader is referred to Subsection 2.4 and references therein for additional details on the subject.

The MATLAB open source toolbox (https://github.com/bayesnet/bnt) was used to compile the network and compute the posterior probabilities. The latter were calculated by resolving the network using a junction tree algorithm, which perform exact inference (Lauritzen \& Spiegelhalter, 1988). Given the limited size of the example proposed here, exact inference was achieved in a few seconds of computation.
More complex or larger BN may need approximate inference methods to resolve the entire network.

In the synthetic failure scenario illustrated here, all three voltage sensors $V_{b}, V_{e}$ and $V_{m}$ indicate anomalous values. The relationship between those voltage sensors and potential faults are visible in Figure 5. Changes in voltage readings at the battery level $V_{b}$ can be caused by $\mathrm{SOC}, \mathrm{SOH}$ or changes in the winding resistance of the motor. Similarly, changes in voltage readings at the ESC and motor level $V_{e}, V_{m}$ can be caused by MOSFET or PWM faults, or again by the winding resistance of the motor.

If all 3 sensors suggests anomalous voltage values, however, the $\mathrm{BN}$ suggests that the most probable cause is the battery SOC, given the direct link to the voltage measurements at the battery level:

$$
\begin{aligned}
\operatorname{Pr}\{\mathrm{SOC} & \left.=1 \mid V_{b}, V_{e}, V_{m}\right\}=0.82021, \\
\operatorname{Pr}\{\mathrm{SOH} & \left.=1 \mid V_{b}, V_{e}, V_{m}\right\}=0.5943, \\
\operatorname{Pr}\left\{\mathrm{TR}=1 \mid V_{b}, V_{e}, V_{m}\right\} & =0.00056469, \\
\operatorname{Pr}\left\{\mathrm{MOSFET}=1 \mid V_{b}, V_{e}, V_{m}\right\} & =0.044391, \\
\operatorname{Pr}\left\{\mathrm{PWM}=1 \mid V_{b}, V_{e}, V_{m}\right\} & =0.45402, \\
\operatorname{Pr}\{\text { bearing } & \left.=1 \mid V_{b}, V_{e}, V_{m}\right\}=0.00049766, \\
\operatorname{Pr}\{\mathrm{M} . \text { Wind. } & \left.=1 \mid V_{b}, V_{e}, V_{m}\right\}=0.50274 .
\end{aligned}
$$

The second most probable cause is the battery $\mathrm{SOH}$, also strictly connected to the voltage. The third most likely cause is a change in winding resistance of the motor, since it affects 
the voltage at all levels (battery, ESC, motor). Therefore, after investigating the charge and the health of the battery, the network aid identification by pointing to the component that most likely affect the voltage of the powertrain system, which is the motor windings.

Finally, a second example shows only two voltage nodes with abnormal values, $V_{e}$ and $V_{m}$. In this case, the obtained posterior failure probabilities are:

$$
\begin{aligned}
\operatorname{Pr}\left\{\mathrm{SOC}=1 \mid V_{e}, V_{m}\right\} & =0.073429, \\
\operatorname{Pr}\left\{\mathrm{SOH}=1 \mid V_{e}, V_{m}\right\} & =0.070601, \\
\operatorname{Pr}\left\{\mathrm{TR}=1 \mid V_{e}, V_{m}\right\} & =7.2931 e-05, \\
\operatorname{Pr}\left\{\mathrm{MOSFET}=1 \mid V_{e}, V_{m}\right\} & =0.2991, \\
\operatorname{Pr}\left\{\mathrm{PWM}=1 \mid V_{e}, V_{m}\right\} & =0.65794, \\
\operatorname{Pr}\left\{\text { bearing }=1 \mid V_{e}, V_{m}\right\} & =6.5254 e-05, \\
\operatorname{Pr}\{\mathrm{M} . \text { Wind. } & \left.=1 \mid V_{e}, V_{m}\right\}=0.073662,
\end{aligned}
$$

The observed failure probabilities with abnormal values observed in $V_{e}$ and $V_{m}$ detect and isolate PWM and MOSFET as most probable fault modes than the others. The reason lies in the connection of the motor winding with the voltage at the battery level, $V_{b}$. Winding resistance affects all three voltage readings; since $V_{e}=1, V_{m}=1$, but $V_{b}=0$, the probability that a change in winding resistance causes the current state of the network is very low, and comparable with the probability that a battery anomaly (SOC or $\mathrm{SOH}$ ) is causing such a state.

Thus the BN-based tool appears to be able to detect and isolate faults based on the their failure probabilities. Though this is not the final version of the proposed tool, and still a workin-progress. As discussed earlier in the Section, with additional information from experiments, SME's, etc, it is possible to further fine tuning the CPTs and introduce quantitative values for the probability of mis-detection and probability of false alarms, thus enhancing the accuracy of the $\mathrm{BN}$ posterior probabilities when a fault is detected.

\section{Conclusions}

This work presented the integration of failure mode and effect analysis (FMEA) with Bayesian networks (BN) to enhance fault detection and isolation approaches for electrical powertrain systems. The methodology does not introduce novel instruments, but rather takes advantage of the synergistic effect of design tools (FMEA) and computer science methods (BNs) to enhance the powertrain system diagnosis.

The $\mathrm{BN}$ is composed of nodes representing faults, failure modes, sensors (or anomaly detectors), and root causes, directly coming from the design FMEA of the system. The challenge, already well known in the field of reliability analysis, is represented by the assessment of the failure probabilities, and the effort to fill conditional probability tables that grow exponentially with the number of ancestors. However, the preliminary analysis presented here suggests that even qualitative assessment of such probabilities can help disambiguate between faults that could be defined equally probable without information from the FMEA and their likelihood of occurrence.

The methodology applied to this specific example focuses on fault isolation in a small electric vehicle powertrain. However, the broader goal of the research is to provide a comprehensive tool to perform anomaly detection, diagnosis and prognosis methodologies to electric aircraft system. This object is supported by experimental activities aimed at refining and testing such diagnostic and prognostic tools.

Next steps of the research include the enhancement of the BN model with multiple rotors, to represent a typical UAV configuration, add fault quantification to asses severity, and eventually perform failure prediction.

\section{ACKNOWLedgments}

This work was supported by the System-Wide Safety (SWS) project under the Airspace Operations and Safety Program within the NASA Aeronautics Research Mission Directorate (ARMD).

\section{REFERENCES}

Abramov, I. V., Nikitin, Y. R., Abramov, A. I., Sosnovichellasosnovich, E. V., \& Bozek, P. (2014). Control and diagnostic model of brushless dc motor. In Journal of electrical engineering.

Awadallah, M. A., \& Morcos, M. M. (2002). Statorwinding fault diagnosis of pm brushless dc motor drives. In IEEE (Ed.), Large engineering systems conference on power engineering (p. 147-152). doi: 10.1109/LESCPE.2002.1020681

Bobbio, A., Portinale, L., Minichino, M., \& Ciancamerla, E. (2001). Improving the analysis of dependable systems by mappping fault trees into bayesian networks. In Reliability engineering and systems safety (Vol. 71, p. 249-260).

Cristea, G., \& Constantinescu, D. (2017, oct). A comparative critical study between FMEA and FTA risk analysis methods. IOP Conference Series: Materials Science and Engineering, 252, 012046.

Daigle, M., \& Kulkarni, C. (2013, October). Electrochemistry-based battery modeling for prognostics. In Annual conference of the prognostics and health management society 2013 (p. 249-261). 
Daigle, M., Sankararaman, S., \& Kulkarni, C. (2015, March). Stochastic prediction of remaining driving time and distance for a planetary rover. In 2015 ieee aerospace conference.

Darmstadt, P., \& et al. (2019, June). Hazards analysis and failure modes and effects criticality analysis (fmeca) of four concept vehicle propulsion systems (Tech. Rep.). NASA.

Gilabert, A. G. E. (2011). Mapping fmea into bayesian networks. International Journal of Performability Engineering, 7(6), 525-537.

Gorospe, G., Kulkarni, C., \& Hogge, E. (2017). A study of the degradation of electronic speed controllers for brushless dc motors. In Asia pacific conference of the prognostics and health management society.

Gorospe, G. E., \& Kulkarni, C. S. (2017). A novel uav electric propulsion testbed for diagnostics and prognostics. In 2017 ieee autotestcon (pp. 1-6).

Hogge, E., Bole, B., Vazquez, S., Kulkarni, C., Strom, T., Hill, B., .. 8, C. Q. (2018). Verification of prognostic algorithms to predict remaining flying time for electric unmanned vehicles. In International journal of prognostics and health management, issn 2153-2648, 2018 021.

Humphreys, M., \& Jacobs, A. (2015). Mixing methods: A bayesian approach. In American political science review.

Kulkarni, C. S., Celaya, J. R., Biswas, G., \& Goebel, K. (2012). Towards a model-based prognostics methodology for electrolytic capacitors: A case study based on electrical overstress accelerated aging. International Journal of Prognostics and Health Management, 5(1), 16.

Kulkarni, C. S., Gorospe, G., Teubert, C., Quach, C., Darafsheh, K., \& Hogge, E. F. (2017). Application of prognostics methodology to virtual laboratory for future aviation and airspace research. In Aiaa modeling and simulation technologies conference. American Institute of Aeronautics and Astronautics.

Larson, G. C. (2015, Dec). Electrical power will change the look of aviation. Air and Space Magazine.

Lauritzen, S. L., \& Spiegelhalter, D. J. (1988). Local computations with probabilities on graphical structures and their application to expert systems. Journal of the Royal Statistical Society: Series B (Methodological),
50(2), 157-194.

Lee, B. H. (2001). Using bayes belief networks in industrial fmea modeling and analysis. In Ieee proceedings of the annual reliability and maintainability symposium.

Medow, M., \& Lucey, C. (2011). A qualitative approach to bayes' theorem. In Bmj evidence-based medicine.

NASA. (2015). Nasa electrified aircraft propulsion (eap) activities.

Pearl, J. (1985). Bayesian networks: A model cf selfactivated memory for evidential reasoning. In 7th conference of the cognitive science society.

Pearl, J. (2000). Causality: models, reasoning and inference. MIT Press Cambridge, MA.

Raymer, D. P. (2018). Aircraft design: A conceptual approach. AIAA.

Rosero, J., Ortega, J., Aldabas, E., \& Romeral, L. (2007, April). Moving towards a more electric aircraft. IEEE Aerospace and Electronic Systems Magazine.

SAE. (2015, Jan). Potential failure mode and effects analysis in design. J173_200901.

\section{BIOGRAPHIES}

Chetan S. Kulkarni is a Research Engineer with KBR Inc, at NASA Ames Research Center, Calif. He received the B.E. (Bachelor of Engineering) degree in Electronics and Electrical Engineering from University of Pune, India in 2002 and the M.S. and Ph.D. degrees in Electrical Engineering from Vanderbilt University, Nashville, TN, in 2009 and 2013, respectively. His current research interests include physicsbased modeling, model-based diagnosis and prognosis for complex systems. Dr. Kulkarni is a member of the Prognostics and Health Management (PHM) Society, SM AIAA and SM IEEE.

Matteo Corbetta is a Research Engineer with KBR Inc, at NASA Ames Research Center, Calif. His research interests include surrogate modeling, model-based and data-driven algorithms for diagnostic and prognostic, and uncertainty quantification methods for autonomous systems. He is a member of the editorial board of the PHM Society, and a member of AIAA and IEEE.

Elinirina I. Robinson is a Research Engineer with KBR Inc, at NASA Ames Research Center, CA. Prior to joining NASA, she received a Ph.D. in Automatic Control in 2018, jointly from ONERA and CNAM (Paris, France), about model-based prognostics for aerospace systems. She received in 2015 an Engineer's Degree in Electrical Engineering from ENSIP (France) and a MSc. in Automatic Control from University of Poitiers (France). Her research interests include diagnostics and prognostics approaches for electrical propulsion systems, 


\begin{tabular}{cc|c} 
Op. Cond & Flight Prof. & $\operatorname{Pr}\{\mathrm{SOC}=1\}$ \\
\hline 0 & 0 & $1 \mathrm{e}-12$ \\
1 & 0 & 0.6 \\
0 & 1 & 0.45 \\
1 & 1 & 0.99 \\
\hline
\end{tabular}

Table 3. Battery SOC. influenced by operational conditions and flight profile.

\begin{tabular}{cc|c} 
Op. Cond & Flight Prof. & $\operatorname{Pr}\{\mathrm{SOH}=1\}$ \\
\hline 0 & 0 & $1 \mathrm{e}-6$ \\
1 & 0 & 0.7 \\
0 & 1 & 0.3 \\
1 & 1 & 0.9 \\
\hline
\end{tabular}

Table 4. Battery SOH. Influenced by operational conditions and flight profile.

filtering and uncertainty propagation methods based on both stochastic and interval analysis algorithms.

\section{APPENDIX}

We report the prior conditional probabilities utilized to produce the results in the paper. They are based on qualitative assumptions by subject matter experts, are for illustration only and should not be considered accurate.

The prior conditional probabilities of root causes: operational conditions, flight profile, internal chemical reactions, and electro-thermal stress, were all set to a default value of 1e-6. Some of the root causes are easily observables (like external temperature, or longer-than-expected flight profile), and therefore their nodes can be set to True when observed. Internal effects like chemical reactions and electro-thermal stresses can only be inferred by the output of the network. To illustrate the approach, we did not discuss likelihood of these internal root causes that cannot be directly measured, hence their prior probability was set to the default value. The likelihood of occurrence of such unobservable root causes, as well as intermediate faults and failures, depends on several factors including design choices, manufacturing processes, initial product defects, and precision of assembly. The prior conditional probabilities of observable variables like voltage, current and temperature depend on sensor properties like sensitivity, accuracy, failure rates, and the thresholds set to define "anomalies", among others.

All tables shows only the probability of observing the node to be True (1), since the probability of the node to be False (0) is the complement to $1, \operatorname{Pr}\{\mathrm{X}=0\}+\operatorname{Pr}\{\mathrm{X}=1\}=1$.

\begin{tabular}{ccc|c} 
Op. Cond & Flight Prof. & Chem. & $\operatorname{Pr}\{\mathrm{TR}=1\}$ \\
\hline 0 & 0 & 0 & $1 \mathrm{e}-15$ \\
1 & 0 & 0 & $1 \mathrm{e}-3$ \\
0 & 1 & 0 & $1 \mathrm{e}-6$ \\
0 & 0 & 1 & 0.8 \\
1 & 1 & 0 & $1 \mathrm{e}-3$ \\
1 & 0 & 1 & 0.9 \\
0 & 1 & 1 & 0.85 \\
1 & 1 & 1 & 0.95 \\
\hline
\end{tabular}

Table 5. Battery thermal runaway. Influenced by operational conditions, flight profile, and internal chemical reaction.

\begin{tabular}{cc|c} 
Op. Cond & el. them. stress & $\operatorname{Pr}\{\mathrm{PWM}=1\}$ \\
\hline 0 & 0 & $1 \mathrm{e}-15$ \\
1 & 0 & $1 \mathrm{e}-3$ \\
0 & 1 & 0.05 \\
1 & 1 & 0.1 \\
\hline
\end{tabular}

Table 6. ESC PWM fault. Influenced by operational conditions and electrical and thermal stresses.

\begin{tabular}{cc|c} 
Op. Cond & ETS & $\operatorname{Pr}\{$ MOSFET $=1\}$ \\
\hline 0 & 0 & $1 \mathrm{e}-15$ \\
1 & 0 & $1 \mathrm{e}-2$ \\
0 & 1 & 0.1 \\
1 & 1 & 0.2 \\
\hline
\end{tabular}

Table 7. ESC MOSFET fault. Influenced by operational conditions and electrical and thermal stresses (ETS).

\begin{tabular}{cc|c} 
Op. Cond & Flight prof. & $\operatorname{Pr}\{\mathrm{B}=1\}$ \\
\hline 0 & 0 & $1 \mathrm{e}-6$ \\
1 & 0 & $1 \mathrm{e}-3$ \\
0 & 1 & $1 \mathrm{e}-5$ \\
1 & 1 & 0.01 \\
\hline
\end{tabular}

Table 8. Motor bearing fault (B). Influenced by operational conditions and flight profile.

\begin{tabular}{cc|c} 
Op. Cond & Flight prof. & $\operatorname{Pr}\{\mathrm{W}=1\}$ \\
\hline 0 & 0 & $1 \mathrm{e}-15$ \\
1 & 0 & $1 \mathrm{e}-3$ \\
0 & 1 & $1 \mathrm{e}-2$ \\
1 & 1 & 0.1 \\
\hline
\end{tabular}

Table 9. Motor winding resistance fault (W). Influenced by operational conditions and flight profile.

\begin{tabular}{ccc|c} 
SOC & SOH & M. Win. & $\operatorname{Pr}\left\{\mathrm{V}_{\mathrm{b}}=1\right\}$ \\
\hline 0 & 0 & 0 & $1 \mathrm{e}-15$ \\
1 & 0 & 0 & 0.9 \\
0 & 1 & 0 & 0.3 \\
0 & 0 & 1 & 0.95 \\
1 & 1 & 0 & 0.1 \\
1 & 0 & 1 & 0.95 \\
0 & 1 & 1 & 0.55 \\
1 & 1 & 1 & 0.99 \\
\hline
\end{tabular}

Table 10. Voltage readings at battery level. Influenced by $\mathrm{SOC}, \mathrm{SOH}$, and motor winding fault. 


\begin{tabular}{l|c} 
SOC, SOH, Bearing, TR, MOSFET & $\operatorname{Pr}\left\{\mathrm{I}_{\mathrm{b}}=1\right\}$ \\
\hline all ancestors False & $1 \mathrm{e}-15$ \\
1 ancestor True & 0.3 \\
2 ancestors True & 0.55 \\
3 ancestors True & 0.7 \\
4 ancestors True & 0.85 \\
5 ancestors True & 0.0 .95 \\
\hline
\end{tabular}

Table 11. Current readings at battery level. Influenced by 5 ancestors: SOC, SOH, motor bearing fault, thermal runaway (TR) and MOSFET fault. The table would have $2^{5}=32$ rows. We have made the simplifying assumption that the probability of observing a current anomalous value depends only on the number of active ancestors, rather than which individual ancestors is activated. By so doing, the prior conditional probabilities to be defined falls from $2^{n}$ to $n+1,6$ in this case).

\begin{tabular}{c|c} 
TR & $\operatorname{Pr}\left\{\mathrm{T}_{\mathrm{b}}=1\right\}$ \\
\hline 0 & $1 \mathrm{e}-15$ \\
1 & 0.999
\end{tabular}

Table 12. Temperature readings at battery level. Caused by thermal runaway (TR).

\begin{tabular}{ccc|c} 
PWM & MOSFET & M. Wind. & $\operatorname{Pr}\left\{\mathrm{V}_{\mathrm{e}}=1\right\}$ \\
\hline 0 & 0 & 0 & $1 \mathrm{e}-15$ \\
1 & 0 & 0 & 0.6 \\
0 & 1 & 0 & 0.6 \\
0 & 0 & 1 & 0.6 \\
1 & 1 & 0 & 0.65 \\
1 & 0 & 1 & 0.65 \\
0 & 1 & 1 & 0.65 \\
1 & 1 & 1 & 0.9 \\
\hline
\end{tabular}

Table 13. Voltage readings at ESC level. Influenced by PWM fault, MOSFET fault, and motor winding resistance.

\begin{tabular}{cc|c} 
MOSFET & Bearing & $\operatorname{Pr}\left\{\mathrm{I}_{\mathrm{e}}=1\right\}$ \\
\hline 0 & 0 & $1 \mathrm{e}-15$ \\
1 & 0 & 0.6 \\
0 & 1 & 0.2 \\
1 & 1 & 0.85 \\
\hline
\end{tabular}

Table 14. Current readings at ESC level. Influenced by MOSFET fault and motor bearing fault.

\begin{tabular}{c|c} 
MOSFET & $\operatorname{Pr}\left\{\mathrm{T}_{\mathrm{e}}=1\right\}$ \\
\hline 0 & $1 \mathrm{e}-15$ \\
1 & 0.9 \\
\hline
\end{tabular}

Table 15. Temperature readings at ESC level. Caused by ESC MOSFET fault.

\begin{tabular}{ccc|c} 
PWM & MOSFET & M. Wind. & $\operatorname{Pr}\left\{\mathrm{V}_{\mathrm{m}}=1\right\}$ \\
\hline 0 & 0 & 0 & $1 \mathrm{e}-3$ \\
1 & 0 & 0 & 0.85 \\
0 & 1 & 0 & 0.87 \\
0 & 0 & 1 & 0.89 \\
1 & 1 & 0 & 0.9 \\
1 & 0 & 1 & 0.93 \\
0 & 1 & 1 & 0.95 \\
1 & 1 & 1 & 0.999
\end{tabular}

Table 16. Voltage readings at motor level. Influenced by ESC PWM and MOSFET faults, as well as motor winding resistance.

\begin{tabular}{ccc|c} 
MOSFET & Bearing & M. Wind. & $\operatorname{Pr}\left\{\mathrm{I}_{\mathrm{m}}=1\right\}$ \\
\hline 0 & 0 & 0 & $1 \mathrm{e}-12$ \\
1 & 0 & 0 & 0.8 \\
0 & 1 & 0 & 0.85 \\
0 & 0 & 1 & 0.85 \\
1 & 1 & 0 & 0.9 \\
1 & 0 & 1 & 0.92 \\
0 & 1 & 1 & 0.92 \\
1 & 1 & 1 & 0.99 \\
\hline
\end{tabular}

Table 17. Current readings at motor level. Influenced by ESC MOSFET fault, motor bearing fault and winding resistance.

\begin{tabular}{cc|c} 
Bearing & M. wind. & $\operatorname{Pr}\left\{\mathrm{T}_{\mathrm{m}}=1\right\}$ \\
\hline 0 & 0 & $1 \mathrm{e}-12$ \\
1 & 0 & 0.85 \\
0 & 1 & 0.9 \\
1 & 1 & 0.99 \\
\hline
\end{tabular}

Table 18. Temperature readings at motor level. Influenced by motor bearing fault and winding resistance. 